\title{
Multi-sensitivity, syndetical sensitivity and the asymptotic average- shadowing property for continuous semi-flows
}

\author{
Risong Li ${ }^{a}, *$, Tianxiu Lu ${ }^{b, c, d}$, Yu Zhao ${ }^{a}$, Hongqing Wang ${ }^{a}$, Haihua Liang ${ }^{a}$ \\ a School of Mathematic and Computer Science, Guangdong Ocean University, Zhanjiang, Guangdong, 524025, People's Republic of \\ China. \\ ${ }^{b}$ Department of Mathematics, Sichuan University of Science and Engineering, Zigong, Sichuan, 643000, People's Republic of China. \\ ${ }^{c}$ Artificial Intelligence Key Laboratory of Sichuan Province, Zigong, Sichuan, 643000, People's Republic of China. \\ ${ }^{d}$ Bridge Non-destruction Detecting and Engineering Computing Key Laboratory of Sichuan Province, Zigong, Sichuan, 643000, \\ People's Republic of China.
}

Communicated by B. Samet

\begin{abstract}
In this paper, for a continuous semi-flow $\theta$ on a compact metric space $E$ with the asymptotic average-shadowing property (AASP), we show that if the almost periodic points of $\theta$ are dense in $E$ then $\theta$ is multi-sensitive and syndetically sensitive. Also, we show that if $\theta$ is a Lyapunov stable semi-flow with the AASP, then the space $E$ is trivial. Consequently, a Lyapunov stable semi-flow with the AASP is minimal. Furthermore, we prove that for a syndetically transitive continuous semi-flow on a compact metric space, sensitivity is equivalent to syndetical sensitivity. As an application, we show that for a continuous semi-flow $\theta$ on a compact metric space E with the AASP, if the almost periodic points of $\varphi$ are dense in $E$ then $\theta$ is syndetically sensitive. Moreover, we prove that for any continuous semi-flow $\theta$ on a compact metric space, it has the AASP if and only if so does its inverse limit $(\widetilde{\mathrm{E}}, \widetilde{\theta})$, and if only if so does its lifting continuous semi-flow $(\widehat{\mathrm{E}}, \widehat{\theta})$. Also, an example which contains two numerical experiments is given. Our results extend some corresponding and existing ones. (C)2017 All rights reserved.
\end{abstract}

Keywords: The asymptotic average-shadowing property, strong ergodicity, minimal point, multi-sensitivity, syndetical sensitivity, Lyapunov stable.

2010 MSC: 54H20, 37B40, 37D45.

\section{Introduction}

It is well-known from [46] that it is very interesting and important in dynamical systems theory to find the minimal set. In [19] we can see a brief summarization on this subject. The research on shadowing theory is a very important topic in dynamical systems and ergodic theory [2, 7, 51, 58, 59]. Up to now, there have been many results on the pseudo-orbit tracing property which were published (see $[2,5,7,10$ $14,19,22,27-30,41,42,44,45,51,58,59])$. It is well-known that this kind of property is closely related to stability and chaos in dynamical systems (see $[51,58,59])$. So, it is an important part of stability in

\footnotetext{
*Corresponding author

Email addresses: gdoulrs@163.com (Risong Li), lubeeltx@163.com (Tianxiu Lu), datom@189.cn (Yu Zhao), wanghq3333@126.com (Hongqing Wang), 1hhlucy@126.com (Haihua Liang)

doi:10.22436/jnsa.010.09.34
} 
dynamical systems and ergodic theory (see $[2,7])$. From the numerical point of view, we can understand it as follows: for a map e (resp. a semi-flow $\theta$ ) with the pseudo orbit tracing property (POTP), the orbits which are generated in the process of numerical computations can completely reflect its real dynamical behavior. In [6] the author introduced the concept of the average shadowing property, which is a good tool to characterize Anosov diffeomorphisms in the chaotic theory (see [43]). Kato [25] showed that every Lyapunov stable (LS) flow with the POTP is minimal. Komuro [26] proved that any equidistant flow with the POTP is minimal. He and Wang [19] obtained that each distal flow with the POTP is minimal. Mai [37] established that all pointwise recurrent flows with the POTP are minimal. Gu and Guo [15] considered the relation between the average shadowing property (ASP) and topological ergodicity for flows, and got that any LS flow with the ASP is topologically ergodic. The authors [16] showed that any continuous flow with the ASP is chain transitive, and any LS continuous flow with the ASP is minimal. In [14] Gu introduced the asymptotic average-shadowing property (AASP) for flows, and investigated the relationships between this property and transitivity for flows. He proved that any flow on a compact metric space is chain transitive if it has the positively (or negatively) AASP and any positively (resp. negatively) LS flow is positively (resp. negatively) topologically transitive provided it has positively (resp. negatively) AASP. Furthermore, he gave two conditions under which any flow is minimal. In [4], the authors proved that for a nonsingular (fixed point free) $\mathrm{C}^{1}$ flow over a smooth closed 3-dimensional manifold $M$ with $\mathrm{H}_{2}(M)=0$, if this flow has a dense orbit then there is an open dense set $\mathrm{N} \subset \mathrm{M}$ satisfying that any knotted periodic orbit which intersects $N$ is a nontrivial prime knot. In [28] the authors related the AASP with other notions in topological dynamics. Kulczycki and Oprocha [29] investigated under what conditions can nontransitivity coexist with the AASP. They also studied the limit shadowing property and its relation to the AASP. There are many chaotic phenomena in the real world. Moreover, there exist lots of important and possible applications of chaotic maps. In [53], by using a nonlinear coupling method the authors discussed master-slave synchronization for the fractional difference equation. The numerical simulation which was given by them shows that the designed synchronization method can effectively synchronize the fractional logistic map, and that the Caputo-like delta derivative is adopted as the difference operator. In [54], the authors presented a discrete fractional logistic map in the left Caputo discrete delta's sense and proved that the new model holds discrete memory. Also, they provided the bifurcation diagrams and numerically illustrated the chaotic behaviors, which means that there exist important applications of this map. In [55], the authors gave fractional Logistic map and fractional Lorenz maps of Riemann-Liouville type and considered the general chaotic behaviors of these maps in comparison with the Caputo one. Furthermore, they designed chaos synchronization according to the stability results. From the obtained numerical results one can see the method's effectiveness and fractional chaotic map's potential role for secure communication. In [23], the fractional chaotic map was applied in physics and engineering to properly treat some real-world phenomena. A shuffling method was given based on the fractional logistic map. Also, an image encryption scheme was presented by using the XOR operation and the security analysis was established. Their results show that the fractional difference order can make the encryption scheme highly secure. In [24], numerical recurrence formulae were presented to investigate the chaotic motion of the famous Duffing system. The new Adomian polynomial was adopted to treat the cubic nonlinear term. By using the numerical simulation of the phase portraits and the Poincare sections, the chaotic behaviors were studied for varied frequencies, damping coefficients and forces. From the results in [24], we can see that the numerical method is reliable to investigate chaotic systems. In [56], by researching on the monotonic properties of the convergence factor established by applying the Fourier transform to the error functions, $\mathrm{Wu}$ and $\mathrm{Wu}$ gave a realiable choice of the Robin parameter in the nonoverlapping case and numerical results, which mean that the analyzed Robin parameter results in satisfactory convergence rate. In [48], Thomine extended the corresponding result to larger classes of observables, with milder smoothness conditions, and to larger classes of dynamical systems, which may no longer be mixing. A special emphasis is given by him to continuous time systems: semi-flows, flows, and $Z^{d_{1}}$-extensions of flows, where $d_{1} \geqslant 0$. The latter generalization is applied to the geodesic flow on $Z^{d_{1}}$-periodic manifolds of negative sectional curvature. In [1], Alves et al. deduced a variational principle for impulsive semiflows 
defined on compact metric spaces. In particular, they generalized the classical notion of topological entropy to their setting of discontinuous semi flows. In [49], Thomine studied $Z^{d_{1}}$-periodic semi-flows, which are versions in continuous time of $Z^{d_{1}}$-extensions of dynamical systems. These systems are defined by an underlying dynamical system, a step time (the time to wait before the system makes a move), and a step function (the displacement in $Z^{d_{1}}$ at each step). He was interested in two statistics related to these semi-flows: the local time, i.e., the time spent in some subset, and the first return time to the origin. He got some partial results under spectral conditions on the transfer operator of the underlying dynamical system. If the underlying dynamics is Gibbs-Markov, and under additional constraints on the step time and step function, he got distributional asymptotics for the local time, and an equivalent of the tail of the first return time. In [38], Marklof explained how entry and return times can be interpreted in terms of stationary point processes and their Palm distribution and generalized the results by Haydn et al. to non-ergodic maps and continuous-time dynamical systems. From [1, 3, 14-17, 34, 38, 48, 49] we can see that the generalizations of the results obtained for continuous maps over a compact metric space to semiflows on a compact metric space and the study on the dynamical properties of semi-flows on a compact metric space are interesting. Inspired by the works of these references, in this paper we introduce and study the AASP for semi-flows which is the semi-flow version of the AASP for continuous selfmaps over a compact metric space, and show that for a continuous semi-flow $\theta: E \times R^{+} \rightarrow E$ with the AASP, if the almost periodic points of $\theta$ are dense in $E$ then $\theta$ is multi-sensitive. Moreover, it is shown that if $\theta$ is a LS semi-flow with the AASP, then the space E is trivial. Consequently, any LS semi-flow with the AASP is minimal. Furthermore, it is proven that for a syndetically transitive continuous semi-flow $\theta$ on a compact metric space $E$, sensitivity is equivalent to syndetical sensitivity. As an application, it is shown that for a continuous semi-flow $\theta$ on a compact metric space $E$ with the AASP, if the almost periodic points of $\theta$ are dense in $E$ then $\theta$ is syndetically sensitive. Consequently, by our results obtained in this paper we can easily see that the semi-flow versions of Theorem 3.1 in [40] and Proposition 1, Theorem 1, and Corollary 1 in [39] are true. Also, Theorem 3.5 is stronger than the semi-flow version of Theorem 3.1 in [40], Lemma 3.4 is stronger than the semi-flow versions of Proposition 3.3 from [40] in the case that $f=g$, and Lemma 3.2 is the semi-flow versions of Lemma 3.2 from [40] in the case that $f=g$. Moreover, inspired by [18] and [20] we show that for any semi-flow $\theta \in C^{0}\left(R^{+} \times E, E\right)$ on a compact metric space $(E, d)$, it has the AASP if and only if so does its inverse limit $(\widetilde{E}, \widetilde{\theta})$, and if and only if so does its lifting continuous semi-flow $(\widehat{E}, \widehat{\theta})$. These two results extend the corresponding results in [18] and [20] to the AASP. Thus, our results extend some existing ones. We also give an example which contains two numerical experiments.

In Section 2, we will recall some concepts. Our results are obtained in Section 3.

\section{Preliminaries}

In this paper, a dynamical system is a given pair $(E, e)$, where $E$ is a compact metric space and $e$ is a continuous self-map on $E$. Let $R^{+}=[0,+\infty)$. For any given metric space $(E, d)$, we define the product metric $d^{\prime}$ on the product space $E \times E$ by $d^{\prime}\left((u, v),\left(u^{\prime}, v^{\prime}\right)\right)=\max \left\{d\left(u, u^{\prime}\right), d\left(v, v^{\prime}\right)\right\}$ for any two points $\left(u, u^{\prime}\right),\left(v, v^{\prime}\right) \in E \times E$. Let $E$ and $F$ be topological spaces. The space of all continuous maps from $E$ into $F$ by $\mathrm{C}^{0}(\mathrm{E}, \mathrm{F})$.

A map $\theta \in C^{0}\left(R^{+} \times E, E\right)$ is called to be a continuous semi-flow if it satisfies that

(1) $\varphi(0, y)=y$ for any $y \in E$;

(2) $\varphi(n, \varphi(m, y))=\varphi(n+m, y)$ for any $y \in E$ and any $n, m \geqslant 0$.

For any given $x \in E$, the set $\left\{\theta(n, y): n \in R^{+}\right\}$is called the orbit of $\theta$ through $y$. For any fixed $\lambda>0$ and any $L>0$, a bi-sequence $\left(\left\{y_{i}\right\}_{j=a}^{b},\left\{t_{j}\right\}_{j=a}^{b-1}\right)(0 \leqslant a<b \leqslant+\infty)$ is called a $(\lambda, L)$-pseudo-orbit of $\theta$ if $t_{j} \geqslant L$ and $d\left(\theta\left(t_{j}, y_{j}\right), y_{j+1}\right) \leqslant \lambda$ for any $a \leqslant j \leqslant b-1$, and a bi-sequence $\left(\left\{y_{j}\right\}_{j=0}^{+\infty},\left\{t_{j}\right\}_{j=0}^{+\infty}\right)$ is called a $(\lambda, L)$-average-pseudo-orbit of $\theta$ if $t_{j} \geqslant L$ for any $j \geqslant 0$ and there is an integer $N>0$ with 


$$
\frac{1}{n} \sum_{j=0}^{n-1} d\left(\theta\left(t_{k+j}, x_{k+j}\right), x_{k+j+1}\right)<\lambda
$$

for any $n \geqslant N$ and any integer $k \geqslant 0$. A $(\lambda, L)$-pseudo-orbit $\left(\left\{y_{j}\right\}_{j=0}^{+\infty},\left\{s_{j}\right\}_{j=0}^{+\infty}\right)$ of $\theta$ is called to be $\eta$-shadowed in average by the orbit of $\theta$ through $y$ if we have

$$
\limsup _{n \rightarrow \infty} \frac{1}{n} \sum_{j=0}^{n-1} \int_{t_{j}}^{t_{j+1}} d\left(\theta(s, y), \varphi\left(s-t_{j}, y_{j}\right)\right) d s<\eta,
$$

where $t_{0}=0, t_{n}=\sum_{j=0}^{n-1} s_{j}$ and $n=1,2, \cdots$.

A semi-flow $\theta \in C^{0}\left(R^{+} \times E, E\right)$ is called to have the ASP if for any $\eta>0$ there is a $\lambda>0$ such that any $(\lambda, 1)$-average-pseudo-orbit of $\theta$ can be $\eta$-shadowed in average by some orbit of $\theta$.

Definition 2.1. A bi-sequence $\left(\left\{y_{j}\right\}_{j=0}^{+\infty},\left\{t_{j}\right\}_{j=0}^{+\infty}\right)$ is said to be an asymptotic L-average-pseudo-orbit of $\theta$ if $t_{j} \geqslant L$ for any integer $j \geqslant 0$, and

$$
\lim _{n \rightarrow \infty} \frac{1}{n} \sum_{j=0}^{n} d\left(\theta\left(t_{j}, y_{j}\right), y_{j+1}\right)=0 \text {. }
$$

Definition 2.2. An asymptotic L-average-pseudo-orbit $\left(\left\{y_{j}\right\}_{j=0}^{+\infty},\left\{s_{j}\right\}_{j=0}^{+\infty}\right)$ of $\theta$ is called to be asymptotically shadowed in average by the orbit of $\theta$ through $y$, if we have

$$
\lim _{n \rightarrow \infty} \frac{1}{n} \sum_{j=0}^{n} \int_{t_{j}}^{t_{j+1}} d\left(\theta(s, y), \varphi\left(s-t_{j}, y_{j}\right)\right) d s=0,
$$

where $t_{0}=0, t_{n}=\sum_{j=0}^{n-1} s_{j}$, and $n=1,2, \cdots$.

Definition 2.3. A semi-flow $\theta \in C^{0}\left(R^{+} \times E, E\right)$ is called to have the AASP if for any asymptotic 1-averagepseudo-orbit of $\theta$ can be asymptotically shadowed in average by some orbit of $\theta$.

A subset $A \subset X$ is called to be an invariant set of $\theta$ or invariant under $\theta$ if $\theta(t, A) \subset A$ for any $t \geqslant 0$.

A subset $A \subset X$ is called a minimal set of $\theta$ if it is nonempty, closed, and invariant under $\theta$ and it does not contain any proper subset with these three properties.

A semi-flow $\theta \in C^{0}\left(R^{+} \times E, E\right)$ is said to be minimal if $E$ is a unique minimal set of $\theta$. As $E$ is compact, $\theta$ is minimal if and only if the orbit of $\theta$ through the point $y$ is dense in $E$ for any given $y \in E$.

A semi-flow $\theta \in C^{0}\left(R^{+} \times E, E\right)$ is said to be LS if for any $\eta>0$, there is a $\lambda>0$ satisfying that $d(u, v)<\lambda(u, v \in E)$ implies that $d(\theta(t, u), \theta(t, v))<\eta$ for any $t \geqslant 0$.

A map e $: E \rightarrow E$ (resp. a semi-flow $\theta: R^{+} \times E \rightarrow E$ ) is said to be topologically transitive (TT) if for any nonempty and open sets $U, V \subset E$, there exists $n \in\{0,1, \cdots\}$ (resp. $t \in R^{+}$) such that $e^{n}(U) \cap V \neq \emptyset$ (resp. $\theta(t, U) \cap V \neq \emptyset$ ). As $E$ is compact, $e$ (resp. $\theta$ ) is TT if and only if there is some $y \in E$ such that the orbit of $e$ (resp. $\theta$ ) through $y$ is dense in $E$.

A map $e: E \rightarrow E$ (resp. a semi-flow $\theta: R^{+} \times E \rightarrow E$ ) is said to be topologically ergodic (TE) (see $[32,33,35])$ if for any two nonempty and open sets $U, V \subset E$,

$$
\limsup _{n \rightarrow \infty} \frac{1}{n}\left|\left\{i \in\{0,1, \cdots, n-1\}: f^{i}(U) \cap V \neq \emptyset\right\}\right|>0
$$

(resp. $\limsup _{t \rightarrow \infty} \frac{1}{t} l\left(\left\{s \in R^{+}: \theta(s, U) \cap V \neq \emptyset\right\} \cap[0, t]\right)>0$ ). 
A set $\mathrm{J} \subset\{0,1, \cdots\}$ (resp. $\mathrm{J} \subset \mathrm{R}^{+}$) is said to be syndetic if there is a positive integer $\mathrm{N}$ such that $[n, n+N] \cap J \neq \emptyset($ resp. $[t, t+N] \cap J \neq \emptyset)$ for any integer $n \geqslant 0$ (resp. any $t \geqslant 0)$.

A map $e: E \rightarrow E$ (resp. a semi-flow $\theta: R^{+} \times E \rightarrow E$ ) is said to be topologically strongly ergodic or syndetically transitive (TSE or ST) if for any two nonempty and open sets $U, V \subset E,\{n \in\{0,1, \cdots\}$ : $\left.e^{\mathfrak{n}}(\mathrm{U}) \cap \mathrm{V} \neq \emptyset\right\}$ (resp. $\left\{\mathrm{s} \in \mathrm{R}^{+}: \theta(\mathrm{s}, \mathrm{U}) \cap \mathrm{V} \neq \emptyset\right\}$ ) is syndetic.

A map $e: E \rightarrow E$ (resp. a semi-flow $\theta: R^{+} \times E \rightarrow E$ ) is said to be topologically weakly mixing (TWM) if $e \times e$ (resp. $\theta \times \theta)$ is topologically transitive, where $\theta \times \theta: R^{+} \times E \times E \rightarrow E \times E$ is defined by $\theta \times \theta(t,(u, v))=(\theta(t, u), \theta(t, v))$ for any $(u, v) \in E \times E$ and any $t \geqslant 0$.

It is easy to see that both TE and TWM imply TT, and TSE implies TE.

For a semi-flow $\theta$ on a metric space $(E, d)$, if there is $\lambda>0$ such that for any $u \in E$ and any neighborhood $U$ of $u$, there exist $v \in U$ and $t \geqslant 0$ with $d(\theta(t, u), \theta(t, v))>\lambda$, then the semi-flow $\theta$ is said to be sensitive.

A flow $\theta$ on a metric space $(E, d)$ is said to be uniformly almost periodic if for any $\eta>0$ there exist $H=H(\eta)>0$ and a countable real number set $\left\{s_{j}: 0 \leqslant j<\infty\right\}$ with $\left\{s_{j}\right\} \cap(s, s+H(\varepsilon)) \neq \emptyset$ for any $s \geqslant 0$ and $d\left(\theta(s, x), \theta\left(s+s_{j}, x\right)\right)<\eta$ for any $x \in E, s \geqslant 0$, and $0 \leqslant j<\infty$.

Let $E$ be a topological space and $\theta: R^{+} \times E \rightarrow E$ be a semi-flow. A point $u \in E$ is called an almost periodic point of $\theta$ if for any neighborhood $U$ of $u$, there is a syndetic set $A \subset R^{+}$with $\theta(t u) \in U$ for any $t \in A$. Denote the set of almost periodic points of $\theta$ by $\operatorname{AP}(\theta)$. It is known that $u \in A P(\theta)$ if and only if it is a minimal point of $\theta$ (that is, it is a point of some minimal set of $\theta$ ).

\section{Main results}

Let $(E, d)$ be a metric space, $\theta$ be a semi-flow on $E$. For $V \subset E$ and $\lambda>0$, denote

$$
\mathrm{N}_{\theta}(\mathrm{V}, \lambda):=\left\{\mathrm{t} \in \mathbf{R}^{+}: \text {there exist } x, y \in \mathrm{V} \text { with } \mathrm{d}\left(\theta_{\mathrm{t}}(\mathrm{x}), \theta_{\mathrm{t}}(\mathrm{y})\right)>\lambda\right\}
$$

(1) $\theta$ is sensitive if there is a constant $\lambda>0$ with $N_{\theta}(V, \lambda) \neq \emptyset$ for any nonempty open subset $V \subset E$;

(2) $\theta$ is cofinitely sensitive if there is a constant $\lambda>0$ with $N_{\theta}(V, \lambda) \supset[t,+\infty)$ for some $t>0$ and any nonempty open subset $\mathrm{V} \subset \mathrm{E}$;

(3) $\theta$ is multi-sensitive if there is a constant $\lambda>0$ with $\bigcap_{i=1}^{k} N_{\theta}\left(V_{i}, \lambda\right) \neq \emptyset$ for each $k \geqslant 1$ and any nonempty open sets $\mathrm{V}_{1}, \mathrm{~V}_{2}, \cdots, \mathrm{V}_{\mathrm{k}} \subset \mathrm{E}$;

(4) $\theta$ is syndetically sensitive if there is a constant $\lambda>0$ such that the set $N_{\theta}(V, \delta)$ is syndetic for any nonempty open set $\mathrm{V} \subset \mathrm{E}$.

It is noted that if $\theta$ is cofinitely sensitive or multi-sensitive or syndetically sensitive then it is sensitive, and that if $\theta$ is cofinitely sensitive then it is multi-sensitive and syndetically sensitive.

Lemma 3.1 ([35]). If a semi-flow $\theta$ on a nontrivial metric space satisfies that $\underbrace{\theta \times \theta \times \cdots \times \theta}_{l}$ is TT for each $l \geqslant 1$, then it is multi-sensitive.

Lemma 3.2. For any semi-flow $\theta \in \mathrm{C}^{0}\left(\mathrm{R}^{+} \times \mathrm{E}, \mathrm{E}\right)$ on a compact metric space $(\mathrm{E}, \mathrm{d}), \theta \times \theta$ has the AASP if and only if so does $\theta$.

Proof. If $\theta$ has the AASP, then, by the definition every asymptotic 1-average-pseudo-orbit of $\theta$ can be asymptotically shadowed in average by some orbit of $\theta$. Let $\left(\left\{\left(x_{i}, y_{i}\right)\right\}_{i=0}^{\infty},\left\{s_{i}\right\}_{i=0}^{\infty}\right)$ be an asymptotic 1average-pseudo-orbit of $\theta \times \theta$. That is, there exists a positive integer $N$ such that

$$
\lim _{n \rightarrow \infty} \frac{1}{n} \sum_{i=0}^{n} d^{\prime}\left((\theta \times \theta)\left(s_{i},\left(x_{i}, y_{i}\right)\right),\left(x_{i+1}, y_{i+1}\right)\right)=0
$$


Hence, we have

$$
\lim _{n \rightarrow \infty} \frac{1}{n} \sum_{i=0}^{n} d\left(\theta\left(s_{i}, x_{i}\right), x_{i+1}\right)=0
$$

and

$$
\lim _{n \rightarrow \infty} \frac{1}{n} \sum_{i=0}^{n} d\left(\theta\left(s_{i}, y_{i}\right), y_{i+1}\right)=0
$$

So, both $\left(\left\{x_{i}\right\}_{i=0}^{\infty},\left\{s_{i}\right\}_{i=0}^{\infty}\right)$ and $\left(\left\{y_{i}\right\}_{i=0}^{\infty},\left\{s_{i}\right\}_{i=0}^{\infty}\right)$ are asymptotic 1-average-pseudo-orbits of $\theta$. Hence, there exist $z_{1}, z_{2} \in \mathrm{E}$ such that

$$
\lim _{n \rightarrow \infty} \frac{1}{n} \sum_{i=0}^{n} \int_{t_{i}}^{t_{i+1}} d\left(\theta\left(s, z_{1}\right), \theta\left(s-t_{i}, x_{i}\right)\right) d s=0
$$

and

$$
\lim _{n \rightarrow \infty} \frac{1}{n} \sum_{i=0}^{n} \int_{t_{i}}^{t_{i+1}} d\left(\theta\left(s, z_{2}\right), \theta\left(s-t_{i}, y_{i}\right)\right) d s=0,
$$

where $t_{0}=0, t_{n}=\sum_{i=0}^{n-1} s_{i}$, and $n=1,2, \cdots$. By (3.1) and (3.2), we get

$$
\lim _{n \rightarrow \infty} \frac{1}{n} \sum_{i=0}^{n-1} \int_{t_{i}}^{t_{i+1}}\left(d\left(\theta\left(s, z_{1}\right), \theta\left(s-t_{i}, x_{i}\right)\right)+d\left(\theta\left(s, z_{2}\right), \theta\left(s-t_{i}, y_{i}\right)\right)\right) d s=0,
$$

where $t_{0}=0, t_{n}=\sum_{i=0}^{n-1} s_{i}$, and $n=1,2, \cdots$. So, we obtain

$$
\lim _{n \rightarrow \infty} \frac{1}{n} \sum_{i=0}^{n} \int_{t_{i}}^{t_{i+1}} d^{\prime}\left((\theta \times \theta)\left(s,\left(z_{1}, z_{2}\right)\right),(\theta \times \theta)\left(s-t_{i},\left(x_{i}, y_{i}\right)\right)\right) d s=0,
$$

where $t_{0}=0, t_{n}=\sum_{i=0}^{n-1} s_{i}$, and $n=1,2, \cdots$. Thus, $\theta \times \theta$ has the AASP.

Similarly, one can prove that if $\theta \times \theta$ has the AASP, then so does $\theta$.

Lemma 3.3. For any semi-flow $\theta \in \mathrm{C}^{0}\left(\mathrm{R}^{+} \times \mathrm{E}, \mathrm{E}\right)$ on a compact metric space $(\mathrm{E}, \mathrm{d}), \theta \times \theta$ is LS if and only if $\theta$ is LS.

Proof. The proof is very easy and is omitted.

Lemma 3.4. For any semi-flow $\theta \in C^{0}\left(R^{+} \times E, E\right)$ on a compact metric space $(E, d), \overline{A P(\theta)}=E$ if and only if $\overline{\mathrm{AP}(\theta \times \theta)}=\mathrm{E} \times \mathrm{E}$.

Proof. Clearly, $\overline{\mathrm{AP}(\theta \times \theta)}=\mathrm{E} \times \mathrm{E}$ means $\overline{\mathrm{AP}(\theta)}=\mathrm{E}$.

Let $\overline{A P(\theta)}=E$. By the definition, $x \in A P(\theta)$ if and only if $\operatorname{Orb} \theta(x)$ is minimal. So, one can quickly deduce to the case that $\theta$ is minimal. It is known that there exists an $(x, y) \in A P(\theta \times \theta)$. One can easily verify that for any $t, s \geqslant 0, \varphi_{t} \times \varphi_{s}$ is a factor map from $\theta \times \theta$ to $\theta \times \theta$. This implies that $(\theta(t, x), \theta(s, y)) \in$ $\operatorname{AP}(\theta \times \theta)$. As $\theta$ is minimal, $\operatorname{Orb} \theta(x)$ and $\operatorname{Orb} \theta(y)$ are dense in $E$. Therefore, for any nonempty and open subsets $U, V \subset E$, there are $t, s \geqslant 0$ with $(\theta(t, x), \theta(s, y)) \in U \times V$. This means $(\theta(t, x), \theta(s, y)) \in$ $(\mathrm{U} \times \mathrm{V}) \cap A \mathrm{P}(\theta \times \theta)$. This shows $\overline{\mathrm{AP}(\theta \times \theta)}=\mathrm{E} \times \mathrm{E}$. 
Theorem 3.5. For any semi-flow $\theta \in \mathrm{C}^{0}\left(\mathrm{R}^{+} \times \mathrm{E}, \mathrm{E}\right)$ on a compact metric space $(\mathrm{E}, \mathrm{d})$, if $\theta$ has the AASP and $\overline{\operatorname{AP}(\theta)}=\mathrm{E}$, then $\theta \times \theta$ is TSE (or ST).

Proof. By Lemmas 3.2 and 3.4, it is enough to show that if $\theta$ has the AASP and $\overline{\operatorname{AP}(\theta)}=\mathrm{E}$, then $\theta$ is TSE (or ST). Let $U$ and $V$ be two nonempty open subsets of $E$. For $a \in E$ and any $t>0$, we write $B(a, t)=\{b \in$ $E: d(a, b)<t\}$. As $\overline{A P(\theta)}=E$, one can choose $x \in U \cap A P(\theta), y \in V \cap A P(\theta)$, and $\varepsilon>0$ with $B(x, \varepsilon) \subset U$ and $B(y, \varepsilon) \subset V$. Since $x, y \in A P(\theta), J_{x}=\left\{r \geqslant 0: \theta_{r}(x) \in B\left(x, \frac{1}{2} \varepsilon\right)\right\}$ and $J_{y}=\left\{r \geqslant 0: \theta_{r}(y) \in B\left(y, \frac{1}{2} \varepsilon\right)\right\}$ are syndetic, which implies that there exist $N_{1}, N_{2} \in \mathbb{N}$ with $\left[t, t+N_{1}\right] \cap J_{x} \neq \emptyset$ and $\left[t, t+N_{2}\right] \cap J_{y} \neq \emptyset$ for any $t \geqslant 0$. Let $N=\max \left\{N_{1}, N_{2}\right\}$. By compactness and continuity, there is $\delta>0$ such that $d(u, v)<\delta(u, v \in X)$ implies $d\left(\theta_{t}(u), \theta_{t}(v)\right)<\frac{1}{2} \varepsilon$ for any $t \in[0, N]$. Write $D=\sup \{d(a, b): a, b \in X\}$.

Since $\theta$ has the AASP, by the definition, every asymptotic 1-average-pseudo-orbit is asymptotic shadowed in average by some point in $E$. Now one can define a bi-sequence sequence $\left(\left\{u_{i}\right\}_{i=0}^{\infty},\left\{t_{i}\right\}_{i=0}^{\infty}\right)$ with $t_{i}=1$ for any integer $i \geqslant 0$ as follows. Let

$$
\begin{aligned}
u_{0} & =x, y=u_{1} \\
u_{2} & =x, y=u_{3} \\
u_{4} & =x, \theta(1, x), \theta(1, y), y=u_{7} \\
& \vdots \\
u_{2^{k}} & =x, \theta(1, x), \cdots, \theta\left(2^{k-1}-1, x\right), \theta\left(2^{k-1}-1, y\right), \cdots, \theta(1, y), y=u_{2^{k+1}-1}
\end{aligned}
$$

It is easily seen that for any $2^{k} \leqslant n<2^{k+1}$,

$$
\frac{1}{n} \sum_{i=0}^{n} d\left(\theta\left(1, u_{i}\right), u_{i+1}\right)<\frac{k+1}{n} D \leqslant \frac{\log _{2} n}{n} D .
$$

So,

$$
\lim _{n \rightarrow \infty} \frac{1}{n} \sum_{i=0}^{n} d\left(\theta\left(1, u_{i}\right), u_{i+1}\right)=0 .
$$

Consequently, $\left(\left\{u_{i}\right\}_{i=0}^{\infty},\left\{t_{i}\right\}_{i=0}^{\infty}\right)$ is an asymptotic 1-average-pseudo-orbit of $\theta$. So, it can be asymptotically shadowed in average by some $u \in E$, that is,

$$
\lim _{n \rightarrow \infty} \frac{1}{n} \sum_{i=0}^{n} \int_{i}^{i+1} d\left(\theta(t, u), \theta\left(t-i, u_{i}\right)\right) d t=0 .
$$

We have the following claim.

Claim. The following hold:

(1) there exist infinite $i \in \mathbb{N}$ such that

$$
u_{i} \in\left\{x, \theta(1, x), \cdots, \theta\left(2^{i}-1, x\right)\right\} \quad \text { and } \quad \int_{i}^{i+1} d\left(\theta(t, u), \theta\left(t-i, u_{i}\right)\right) d t<\delta
$$

(2) there exist infinite $i \in \mathbb{N}$ such that

$$
u_{i} \in\left\{y, \theta(1, y), \cdots, \theta\left(2^{i}-1, y\right)\right\} \quad \text { and } \quad \int_{i}^{i+1} d\left(\theta(t, u), \theta\left(t-i, u_{i}\right)\right) d t<\delta
$$


Proof of Claim. Without loss of generality, we only prove (1). If there exists $M \in \mathbb{N}$ such that for all $i \geqslant M$, if

$$
u_{i} \in\left\{x, \theta(1, x), \cdots, \theta\left(2^{i}-1, x\right)\right\}
$$

then

$$
\int_{i}^{i+1} d\left(\theta(t, u), \theta\left(t-i, u_{i}\right)\right) d t \geqslant \delta
$$

This implies that

$$
\lim _{n \rightarrow \infty} \frac{1}{n} \sum_{i=0}^{n} \int_{i}^{i+1} d\left(\theta(t, u), \theta\left(t-i, u_{i}\right)\right) d t \geqslant \frac{1}{2} \delta .
$$

It is a contradiction.

By Claim, there exist $i_{0}>N, k_{0} \in\left[0,2^{i_{0}}-1\right], j_{0}>i_{0}+N$, and $l_{0} \in\left[0,2^{j_{0}}-1\right]$ such that

$$
u_{i_{0}}=\theta\left(k_{0}, x\right), \quad \int_{i_{0}}^{i_{0}+1} d\left(\theta(t, u), \theta\left(t-i_{0}, u_{i_{0}}\right)\right) d t<\delta
$$

and

$$
u_{j_{0}}=\theta\left(l_{0}, y\right), \quad \int_{j_{0}}^{j_{0}+1} d\left(\theta(t, u), \theta\left(t-j_{0}, u_{j_{0}}\right)\right) d t<\delta
$$

This implies that

$$
u_{i_{0}}=\theta\left(k_{0}, x\right), d\left(\theta\left(t_{i_{0}}, u\right), \theta\left(t_{i_{0}}-i_{0}, u_{i_{0}}\right)\right)<\delta
$$

for some $t_{i_{0}} \in\left[i_{0}, i_{0}+1\right]$ and

$$
u_{j_{0}}=\theta\left(l_{0}, y\right), d\left(\theta\left(t_{j_{0}}, u\right), \theta\left(t_{j_{0}}-j_{0}, u_{j_{0}}\right)\right)<\delta
$$

for some $t_{j_{0}} \in\left[j_{0}, j_{0}+1\right]$.

As $\left[k_{0}+1, k_{0}+1+N\right] \cap J_{x} \neq \emptyset$ and $\left[l_{0}+1, l_{0}+1+N\right] \cap J_{y} \neq \emptyset$, there exist $k^{\prime}, l^{\prime} \in[0, N]$ such that

$$
\theta_{\mathrm{k}_{0}+1+\mathrm{k}^{\prime}}(x) \in \mathrm{B}\left(\mathrm{x}, \frac{1}{2} \varepsilon\right) \text { and } \theta_{\mathrm{l}_{0}+1+\mathrm{l}^{\prime}}(\mathrm{y}) \in \mathrm{B}\left(\mathrm{y}, \frac{1}{2} \varepsilon\right) .
$$

This means that

$$
\theta_{k_{0}+k}(x) \in B\left(x, \frac{1}{2} \varepsilon\right) \text { and } \theta_{l_{0}+l}(y) \in B\left(y, \frac{1}{2} \varepsilon\right),
$$

where $k=1+k^{\prime}$ and $l=1+l^{\prime}$. By (3.3) and (3.4), we get

$$
d\left(\theta_{t_{i_{0}}+k-\left(t_{i_{0}}-i_{0}\right)}(u), \theta_{k_{0}+t_{i_{0}}-i_{0}+k-\left(t_{i_{0}}-i_{0}\right)}(x)\right)<\frac{1}{2} \varepsilon
$$

and

$$
d\left(\theta_{t_{j_{0}}+l-\left(t_{j_{0}}-j_{0}\right)}(u), \theta_{l_{0}+t_{j_{0}}-j_{0}+l-\left(t_{j_{0}}-j_{0}\right)}(y)\right)<\frac{1}{2} \varepsilon .
$$

That is,

$$
\mathrm{d}\left(\theta_{\mathrm{i}_{0}+\mathrm{k}}(\mathrm{u}), \theta_{\mathrm{k}_{0}+\mathrm{k}}(\mathrm{x})\right)<\frac{1}{2} \mathcal{\varepsilon} \quad \text { and } \quad \mathrm{d}\left(\theta_{\mathrm{j}_{0}+\mathrm{l}}(\mathrm{u}), \theta_{\mathrm{l}_{0}+\mathrm{l}}(\mathrm{y})\right)<\frac{1}{2} \varepsilon \text {. }
$$

So, we have

$$
\theta_{i_{0}+k}(u) \in B(x, \varepsilon) \subset U \text { and } \quad \theta_{j_{0}+l}(u) \in B(y, \varepsilon) \subset V .
$$


Let $n_{0}=j_{0}+l-i_{0}-k>0$. Then $u \cap \theta_{n_{0}}^{-1}(V) \neq \emptyset$. This means that $\theta$ is topologically transitive. Let $\mathrm{Q}=\mathrm{U} \cap \theta_{\mathrm{n}_{0}}^{-1}(\mathrm{~V})$. Then there is $\mathrm{p} \in \mathrm{AP}(\theta) \cap \mathrm{Q}$. Write $\mathrm{J}=\{\mathrm{t} \geqslant 0: \theta(\mathrm{t}, \mathrm{p}) \in \mathrm{Q}\}$. By [49], J is syndetic. Clearly, $Q \cap \theta_{\mathrm{r}}^{-1}(\mathrm{Q}) \neq \emptyset$ for all $\mathrm{r} \in \mathrm{J}$. This implies

$$
\emptyset \neq \mathrm{U} \cap \theta_{\mathrm{n}_{0}}^{-1}(\mathrm{~V}) \cap \theta_{\mathrm{r}}^{-1}\left(\mathrm{U} \cap \theta_{\mathrm{n}_{0}}^{-1}(\mathrm{~V})\right) \subset \mathrm{U} \cap \theta_{\mathrm{n}_{0}+\mathrm{r}}^{-1}(\mathrm{~V})
$$

Therefore,

$$
\mathrm{N}_{\theta}(\mathrm{U}, \mathrm{V}) \supset\left\{\mathrm{n}_{0}+\mathrm{r}: \mathrm{r} \in \mathrm{J}\right\} \text {. }
$$

Consequently, $\mathrm{N}_{\theta}(\mathrm{U}, \mathrm{V})$ is syndetic. As $\mathrm{U}, \mathrm{V}$ are arbitrary, $\theta$ is TSE or ST.

Theorem 3.6. For any semi-flow $\theta \in \mathrm{C}^{0}\left(\mathrm{R}^{+} \times \mathrm{E}, \mathrm{E}\right)$ on a compact metric space $(\mathrm{E}, \mathrm{d})$, if $\theta$ has the AASP and $\overline{\mathrm{AP}(\theta)}=\mathrm{E}$, then it is multi-sensitive.

Proof. By Lemmas 3.2 and 3.4, and Theorem 3.5 and its proof, $\underbrace{\theta \times \theta \times \cdots \times \theta}_{\mathrm{m}}$ is TT for any $\mathrm{m} \geqslant 1$. By Lemma 3.1, $\theta$ is multi-sensitive.

Theorem 3.7. For any LS semi-flow $\theta \in \mathrm{C}^{0}\left(\mathrm{R}^{+} \times \mathrm{E}, \mathrm{E}\right)$ on a nontrivial and compact metric space $(\mathrm{E}, \mathrm{d})$, if $\overline{\mathrm{AP}(\theta)}=\mathrm{E}$, then it does not have the AASP.

Proof. If $\theta$ has the AASP, then it is multi-sensitive by Theorem 3.6. This is a contradiction.

In [15], the authors established that any LS flow with the ASP on a compact metric space is TE. For continuous semi-flows, we get the following similar result.

Theorem 3.8. For any $L S$ semi-flow $\theta \in \mathrm{C}^{0}\left(\mathrm{R}^{+} \times \mathrm{E}, \mathrm{E}\right)$ on a compact metric space $(\mathrm{E}, \mathrm{d})$, if it has the AASP, then it is TE.

Proof. By using the argument similar to the proof of Theorem 1 in [15] one can easily obtain the proof of Theorem 3.8.

The authors [15] showed that for any LS flow $\theta$ on a compact metric space E, if it has the ASP, then it is a uniformly almost periodic minimal flow. However, For continuous semi-flows, we obtain the following corollary.

Corollary 3.9. For any $L S$ semi-flow $\theta \in \mathrm{C}^{0}\left(\mathrm{R}^{+} \times \mathrm{E}, \mathrm{E}\right)$ on a compact metric space $(\mathrm{E}, \mathrm{d})$, if it has the AASP, then it is a uniformly almost periodic minimal flow. Consequently, such a semi-flow is TSE.

Proof. The proof is directly obtained from Theorem 3.2 of [17], Theorem 3.4 and Lemma 2.1 in [57].

Corollary 3.10. Any $L S$ semi-flow $\theta \in \mathrm{C}^{0}\left(\mathrm{R}^{+} \times \mathrm{E}, \mathrm{E}\right)$ on a nontrivial and compact metric space $(\mathrm{E}, \mathrm{d})$ does not have the AASP.

Proof. By Corollary 3.9, Theorem 3.7, and Lemma 3.3, this corollary is true.

Remark 3.11. Corollary 3.10 shows that if $\theta \in C^{0}\left(R^{+} \times E, E\right)$ is an LS semi-flow with the AASP on a compact metric space $E$, then this space $E$ is trivial.

Proposition 3.12. For any sensitive semi-flow $\theta \in C^{0}\left(R^{+} \times E, E\right)$ on a compact metric space $(E, d)$, if $\overline{A P(\theta)}=E$, then it is syndetically sensitive.

Proof. Suppose that $\theta$ is sensitive with sensitivity constant $\delta>0$, and that $V \subset E$ is nonempty and open. Let $l \in N_{\varphi}(V, \delta)$. As $\overline{A P(\theta)}=E$, by Lemma 3.4, $\overline{A P(\theta \times \theta)}=E \times E$. So, one may choose $y, z \in V$ with $(y, z) \in A P(\theta \times \theta)$ and $d\left(\theta_{l}(y), \theta_{l}(z)\right)>\delta$. Let $U, Q \subset E$ be small enough open sets such that $\theta_{l}(y) \in U$, $\theta_{\mathrm{l}}(z) \in \mathrm{Q}$ and $\mathrm{d}(\mathrm{u}, w)>\delta$ for any $\mathrm{u} \in \mathrm{U}$, and any $w \in \mathrm{Q}$. It is clear that $\mathrm{U} \times \mathrm{Q}$ is nonempty and open, and that $\left(\theta_{l}(y), \theta_{l}(z)\right) \in U \times Q$. Hence, $N_{\theta}\left(\left(\theta_{l}(y), \theta_{l}(z)\right), U \times Q\right)$ is syndetic. By the above argument, we can see that $N_{\theta}\left(\left(\theta_{l}(y), \theta_{l}(z)\right), U \times Q\right)+l \subset N_{\theta}(V, \delta)$. Consequently, $N_{\theta}(V, \delta)$ is syndetic. This implies $\theta$ is syndetically sensitive. 
Remark 3.13. Proposition 3.12 extends Proposition 1 in [45] to continuous semi-flows.

Proposition 3.14. For any sensitive semi-flow $\theta \in \mathrm{C}^{0}\left(\mathrm{R}^{+} \times \mathrm{E}, \mathrm{E}\right)$ on a compact metric space $(\mathrm{E}, \mathrm{d})$, if it is $S T$ but not minimal, then it is syndetically sensitive.

Proof. Let $b \in E$ be such that of $\overline{\mathrm{O}_{\theta}(\mathrm{b})}=\overline{\left\{\theta_{\mathrm{t}}(\mathrm{b}): t \geqslant 0\right\}} \neq \mathrm{E}$. Let $\mathrm{c} \in\left(\mathrm{E} \backslash \overline{\mathrm{O}_{\varphi}(\mathrm{b})}\right)$, and set $\delta=$ $\frac{1}{5} \mathrm{~d}\left(\mathrm{c}, \overline{\mathrm{O}_{\varphi}(\mathrm{b})}\right)>0$. Write $\mathrm{V}=\mathrm{B}(\mathrm{c}, \delta)$. By the definition, for any nonempty open set $\mathrm{U} \subset \mathrm{E}, \mathrm{N}_{\theta}(\mathrm{U}, \delta)$ is syndetic with some bound $M_{1} \in\{1,2, \cdots\}$ for the gaps. Choose an open subset $Q \subset E$ such that $b \in Q$ and $x \in Q$ imply that $d\left(\theta_{i}(b), \theta_{i}(x)\right)<\delta$ for any $i \in\left\{0,1, \cdots, M_{1}\right\}$. It is easily seen that $d\left(\theta_{i}(Q), V\right) \geqslant 2 \delta$ for any $i \in\left\{0,1, \cdots, M_{1}\right\}$. By hypothesis, $N_{\theta}(U, Q)$ is syndetic with some bound $M_{2} \in\{1,2, \cdots\}$ for the gaps. For any $t \geqslant 0$, we can choose $j \in\left\{1,2, \cdots, M_{2}\right\}$ and $u \in U$ with $\theta_{t+j}(u) \in Q$. By the choice of $Q$, we have $d\left(\theta_{t+j+i}(u), V\right) \geqslant 2 \delta$ for any $i \in\left\{1,2, \cdots, M_{1}\right\}$. Take $i \in\left\{1,2, \cdots, M_{1}\right\}$ and $u^{\prime} \in U$ with $\theta_{t+j+i}(u) \in V$. Then $d\left(\theta_{t+j+i}(u), \theta_{t+j+i}\left(u^{\prime}\right)\right) \geqslant 2 \delta>\delta$. As $j+i \leqslant M_{1}+M_{2}, N_{\theta}(u, \delta)$ is a syndetic set with $M_{1}+M_{2}$ as a bound for the gaps.

Remark 3.15. Proposition 3.14 extends Theorem 1 in [39] to continuous semi-flows.

Corollary 3.16. For a ST continuous semi-flow on a compact metric space $(\mathrm{E}, \mathrm{d})$, sensitivity is equivalent to syndetical sensitivity.

Proof. It follows from Propositions 3.12 and 3.14.

Remark 3.17. Corollary 3.16 extends Corollary 1 in [39] to continuous semi-flows.

Theorem 3.18. For any semi-flow $\theta \in \mathrm{C}^{0}\left(\mathrm{R}^{+} \times \mathrm{E}, \mathrm{E}\right)$ on a compact metric space $(\mathrm{E}, \mathrm{d})$, if it has the AASP and $\overline{\mathrm{AP}(\theta)}=\mathrm{E}$, then $\theta$ is syndetically sensitive.

Proof. By Theorem 3.6 and Corollary 3.16, this theorem holds.

Let $\theta \in C^{0}\left(R^{+} \times E, E\right)$ on a compact metric space $(E, d)$ be a semi-flow such that $\theta_{t}$ is an onto map for any $t \geqslant 0$ and $R=(-\infty,+\infty)$. Write

$$
\widetilde{\mathrm{E}}=\left\{\widetilde{x}=\left(x^{\mathrm{t}}\right)_{\mathrm{t} \in \mathrm{R}}: x^{\mathrm{t}} \in \mathrm{E}, x^{\mathrm{t}}=\theta_{\mathrm{t}-\mathrm{s}}\left(x^{\mathrm{s}}\right), \mathrm{s} \leqslant \mathrm{t}\right\} .
$$

Define a metric $\widetilde{d}$ on the space $\widetilde{E}$ by

$$
\widetilde{d}(\widetilde{x}, \widetilde{y})=\int_{-\infty}^{+\infty} e^{-|t|} d\left(x^{t}, y^{t}\right) d t
$$

for any $\widetilde{x}, \widetilde{y} \in \widetilde{E}$. Let $\widetilde{\theta}: R \times \widetilde{E} \rightarrow \widetilde{E}$ be defined as $\widetilde{\theta}_{t}(\widetilde{x})=\widetilde{\theta}(t, \widetilde{x})=\left(x^{t+s}\right)_{s \in R}$ for any $\widetilde{x} \in \widetilde{E}$ and any $t \in R$. Clearly, $\widetilde{\theta} \in C^{0}(R \times \widetilde{E}, \widetilde{E})$. The flow $(\widetilde{E}, \widetilde{\theta})$ is called the inverse limit of the semi-flow $(E, \theta)$. Define a map $\pi: \widetilde{E} \rightarrow E$ by $\pi\left(\left(x^{t}\right)_{t \in R}\right)=x^{0}$ for any $\left(x^{t}\right)_{t \in R} \in \widetilde{E}$. Then $\pi$ is a continuous onto map with $\pi \circ \widetilde{\theta}=\theta_{t}$ for any $t \geqslant 0$. The following lemma is needed.

Lemma 3.19 ([52]). Let $\mathbb{N}_{0}=\{0,1,2, \cdots\}$ and $\left\{b_{i}\right\}_{i=0}^{\infty} \subset \mathbb{N}_{0}$ be a bounded sequence. Then

$$
\lim _{m \rightarrow \infty} \frac{1}{m} \sum_{j=0}^{m-1} b_{j}=0
$$

if and only if there is a subset $S \subset \mathbb{N}_{0}$ with density zero (that is, $\lim _{\mathfrak{m}} \frac{1}{m} \cdot \operatorname{Card}(S \cap\{0,1, \cdots, m-1\})=0$ ) such that $\lim _{\mathrm{m} \notin \mathrm{S}, \mathrm{m} \rightarrow \infty} \mathrm{b}_{\mathrm{m}}=0$ where $\operatorname{Card}(\mathrm{A})$ is the cardinality of a set $\mathrm{A}$.

Remark 3.20. According to [47], we can only consider the pseudo orbits $\left(\left(t_{i}, x_{i}\right)_{i=0}^{\infty}\right)$ of $\theta$ with $1 \leqslant t_{i}<2$ for every integer $i \geqslant 0$ when we study the shadowing property of a semi-flow or flow $\theta$ on a metric space $(E, d)$. So, for the AASP, one can only consider the asymptotic-average pseudo orbits $\left(\left(t_{i}, x_{i}\right)_{i=0}^{\infty}\right)$ of $\theta$ with $1 \leqslant t_{i}<2$ for every integer $i \geqslant 0$. 
From Theorem 2.7 in [20] we know that a semi-flow $\theta \in C^{0}\left(R^{+} \times E, E\right)$ on a compact metric space $(E, d)$ has the shadowing property if and only if so does its inverse limit $(\widetilde{E}, \widetilde{\theta})$. For the AASP and continuous semi-flow, we have the following result.

Theorem 3.21. For any semi-flow $\theta \in \mathrm{C}^{0}\left(\mathrm{R}^{+} \times \mathrm{E}, \mathrm{E}\right)$ on a compact metric space $(\mathrm{E}, \mathrm{d})$, it has the AASP if and only if so does its inverse limit $(\widetilde{\mathrm{E}}, \widetilde{\theta})$.

Proof. By Remark 3.20, Lemma 3.19, and the method used in the proof of Theorem 2.7 in [20], the proof of Theorem 3.21 is easily obtained and is omitted.

Form the proofs of Proposition and Theorem 1 in [18] one can see that a semi-flow $\theta \in C^{0}\left(R^{+} \times E, E\right)$ on a compact metric space $(E, d)$ has the shadowing property if and only if so does its lifting continuous semi-flow. For the notions of a lifting continuous semi-flow and a projecting continuous semi-flow we refer to [18]. For the AASP, we have a similar result (see Theorem 3.22).

Theorem 3.22. For any semi-flow $\theta \in \mathrm{C}^{0}\left(\mathrm{R}^{+} \times \mathrm{E}, \mathrm{E}\right)$ on a compact metric space $(\mathrm{E}, \mathrm{d})$, it has the AASP if and only if so does its lifting continuous semi-flow $(\widehat{\mathrm{E}}, \widehat{\theta})$.

Proof. The proof of Theorem 3.22 is similar to that of Theorem 1 in [18] and is omitted.

Remark 3.23. Similarly, one can prove the results in this paper are valid for the average-shadowing property on continuous semi-flows.

Let a self-map $f$ of a compact metric space $(E, d)$ be continuous. One can define an equivalence relation $\sim$ in the product space $[0,1] \times E$ as follows (see [21]).

For any $\left(s_{1}, x_{1}\right),\left(s_{2}, x_{2}\right) \in[0,1] \times E,\left(s_{1}, x_{1}\right) \sim\left(s_{2}, x_{2}\right)$ if and only if one of the following conditions hold.

(1) $\left(s_{1}, x_{1}\right)=\left(s_{2}, x_{2}\right)$;

(2) $s_{1}=1, s_{2}=0$ and $x_{2}=g\left(x_{1}\right)$.

Let $W=([0,1] \times E) / \sim$. By [8], $W$ is compact and metrizable. Then the suspended semi-flow $\varphi(f)$ induced by it on $W$ is defined as $\varphi(f)(a,[(b, x)])=\left[a+b-m, f^{m}(x)\right]$ for any $a \geqslant 0$ and any $[(b, x)] \in W$ with $m \leqslant a+b<m+1$ and $m \in\{0,1,2, \cdots\}$. In [34] we proved that a continuous self-map of a compact metric space is chaotic in the sense of Devaney if and only if so is its suspended semi-flow, and that a continuous self-map of a compact metric space is mixing (in the sense of statistics) if and only if so is its suspended semi-flow.

Lemma 3.24. A continuous self-map of a compact metric space has the AASP if and only if so does its suspended semi-flow.

Proof. By the definitions and the method in [21], the proof is easily obtained and is omitted.

Lemma 3.25. A continuous self-map of a compact metric space is multi-sensitive or syndetically sensitive or cofinetly sensitive if and only if so does its suspended semi-flow.

Proof. By the definitions and the proof of Theorem 2.2 from [21], we easily obtain the proof of this lemma.

Example 3.26. Let $f:[0,1] \rightarrow[0,1]$ be the tent map defined by $f(x)=1-|1-2 x|$ for any $x \in[0,1]$. Then the map and its suspended semi-flow has the AASP. Consequently, its suspended semi-flow have all the properties which are discussed in this paper. So, by Lemmas 3.24 and 3.25 we can see the chaotic complexities of this suspended semi-flow by the chaotic complexities and one numerical experiment of the tent map. 
In fact, for every continuous self-map on the unit interval, it is well-known from [9] that if it is topologically mixing, then it has the specification property. Since it is well-known that the tent map is topologically mixing, it has the specification property. By Theorem 3.8 in [28], the tent map has the AASP. By Lemma 3.24, its suspended semi-flow has the AASP. Also, we know that the tent map is sensitive. By Theorem 2 in [39], the tent map is cofinitely sensitive. Consequently, it is multi-sensitive and syndetically sensitive. The chaotic figure of the iterative system $x_{n+1}=f\left(x_{n}\right)$ for any $n \in\{0,1, \cdots\}$ is in Fig. 2 from [31] for the tent map $f$, where the initial value is taken at random, say, $x_{0}=0.7851$, the iterative times $n=1000$. So, Fig. 2 from [31] shows that the chaotic figure of the tent map f. For the figure of the tent map, we can see Fig. 1 from [31]. Let $Q(X)=\frac{1}{2}-4 x^{2}$ for any $x \in\left[-\frac{1}{2}, \frac{1}{2}\right]$. By [50], the logistic map $F(x)=4 x(1-x)$ is topologically conjugate to $Q(X)$. We know that the logistic map $F(x)=4 x(1-x)$ is topologically conjugate to the tent map $f$. Choose $Q\left(x_{0}\right)=0.41857322657198$ and $Q\left(x^{\prime}\right)=0.41857322657197$ which have only $10^{-14}$ different to test the sensitivity. The result is shown in Table 1 from [50]. This also shows that $Q(X)$ has very strong chaotic property which implies the tent map $f$ has very strong chaotic property. Moreover, we can easily show that $\frac{1}{2}$ is a sensitive constant of the tent map with respect to cofinite sensitivity. Therefore, by the definition, for any $\delta \in\left(0, \frac{1}{2}\right), \delta$ is a sensitive constant of the tent map with respect to cofinite sensitivity. Now, we take $x_{0}=0.00001$ and $x_{1}=0.000011$. Then $f^{8}\left(x_{0}\right)=0.00256$, $f^{8}\left(x_{1}\right)=0.002816, f^{15}\left(x_{0}\right)=0.32768, f^{15}\left(x_{1}\right)=0.360448, f^{16}\left(x_{0}\right)=0.65536, f^{16}\left(x_{1}\right)=0.720896, f^{17}\left(x_{0}\right)=$ $0.68928, f^{17}\left(x_{0}\right)=0.558208, f^{18}\left(x_{0}\right)=0.62144, f^{18}\left(x_{1}\right)=0.883584, f^{19}\left(x_{0}\right)=0.75712$, and $f^{19}\left(x_{1}\right)=0.232832$. Consequently, $\left|f^{19}\left(x_{0}\right)-f^{19}\left(x_{1}\right)\right|>\frac{1}{2}$. Let $x_{2}=0.5$ and $x_{3}=0.5000001$. Then $f\left(x_{2}\right)=0, f\left(x_{3}\right)=0.9999998$, $f^{2}\left(x_{2}\right)=0, f^{2}\left(x_{3}\right)=0.0000004, f^{3}\left(x_{2}\right)=0, f^{3}\left(x_{3}\right)=0.0000008, f^{16}\left(x_{2}\right)=0, f^{16}\left(x_{3}\right)=0.0065536, f^{22}\left(x_{2}\right)=0$, $f^{22}\left(x_{3}\right)=0.4194304$, and $f^{23}\left(x_{2}\right)=0, f^{23}\left(x_{3}\right)=0.8388608$. So, $\left|f^{23}\left(x_{2}\right)-f^{23}\left(x_{3}\right)\right|>\frac{1}{2}$. By the definitions and the proof of Theorem 2.2 from [21], we can see that $\delta>0$ is a sensitive constant of $f$ with respect to the corresponding sensitive forms if and only if $\delta>0$ is a sensitive constant of the suspended semi-flow $\varphi(f)$ induced by $f$ with respect to the same sensitive forms. Thus, the above proof and numerical experiments can show the tent map $f$ and the suspended semi-flow $\varphi(f)$ induced by $f$ have all the properties which are discussed in this article.

\section{Conclusion}

From $[3,14-17,20,34,36]$ we can see that research on chaotic properties in continuous semi-flows is very difficult. Especially, we know very little about various shadowing properties of semi-flows. In this paper we define and consider the AASP for semi-flows which is the semi-flow version of the AASP for continuous self-maps over a compact metric space, and show that for a continuous semi-flow $\theta$ : $\mathrm{E} \times \mathrm{R}^{+} \rightarrow \mathrm{E}$ with the AASP, if the almost periodic points of $\theta$ are dense in $\mathrm{E}$ then $\theta$ is multi-sensitive. This is a very strong result. Also, it is proven that if $\theta$ is an LS semi-flow with the AASP, then the space $\mathrm{E}$ is trivial. This means that any LS semi-flow with the AASP is minimal. Moreover, it is proven that for a syndetically transitive continuous semi-flow $\theta$ on a compact metric space $E$, sensitivity is equivalent to syndetical sensitivity. As an application, we show that for a continuous semi-flow $\theta$ on a compact metric space $E$ with the AASP, if the almost periodic points of $\theta$ are dense in $E$ then $\theta$ is syndetically sensitive. Thus, from our results obtained in this paper we can easily see that the semi-flow versions of Theorem 3.1 in [40] and Proposition 1, Theorem 1 and Corollary 1 in [39] are true. Also, Theorem 3.5 is stronger than the semi-flow version of Theorem 3.1 in [40], Lemma 3.4 is stronger than the semi-flow versions of Proposition 3.3 from [40] in the case that $f=g$, and Lemma 3.2 is the semi-flow versions of Lemma 3.2 from [40] in the case that $f=g$. Furthermore, motivated by [18] and [20] we show that for any semi-flow $\theta \in C^{0}\left(R^{+} \times E, E\right)$ on a compact metric space $(E, d)$, it has the AASP if and only if so does its inverse limit $(\widetilde{E}, \widetilde{\theta})$, and if only if so does its lifting continuous semi-flow $(\widehat{E}, \widehat{\theta})$. These two results extend the corresponding results in [18] and [20] to the AASP. Consequently, our main results extend some existing ones. We also present an example which contains two numerical experiments. Note that the shadowing property is closely related to chaos. In the near future we will continue to analyze and study other chaotic properties in continuous semi-flows. 


\section{Acknowledgment}

The authors are very grateful to the referees for their careful reading, significant comments and suggestions, which helped us improve this paper.

This research was supported by the Project of Enhancing School With Innovation of Guangdong Ocean University (Grant NO. GDOU2016050207), the Key Scientific and Technological Research Project of Science and Technology Department of Zhanjiang City (Grant 2010C3112005), the National Natural Science Foundation of China (11501391), the Opening Project of Artificial Intelligence Key Laboratory of Sichuan Province (2015RZJ01) and the Opening Project of Bridge Non-destruction Detecting and Engineering Computing Key Laboratory of Sichuan Province (2014QZJ02).

\section{References}

[1] J. F. Alves, M. Carvalho, C. H. Vásquez, A variational principle for impulsive semiflows, J. Differential Equations, 259 (2015), 4229-4252. 1

[2] N. Aoki, K. Hiraide, Topological theory of dynamical systems, Recent advances, North-Holland Mathematical Library, North-Holland Publishing Co., Amsterdam, (1994). 1

[3] A. Arbieto, R. Ribeiro, Flows with the (asymptotic) average shadowing property on three-dimensional closed manifolds, Dyn. Syst., 26 (2011), 425-432. 1, 4

[4] W. Basener, M. C. Sullivan, Periodic prime knots and topologically transitive flows on 3-manifolds, Topology Appl., 153 (2006), 1236-1240. 1

[5] A. Bielecki, J. Ombach, Dynamical properties of a perceptron learning process: structural stability under numerics and shadowing, J. Nonlinear Sci., 21 (2011), 579-593. 1

[6] M. Blank, Small perturbations of chaotic dynamical systems, (Russian); translated from Uspekhi Mat. Nauk, 44 (1989), 3-28, Russian Math. Surveys, 44 (1989), 1-33. 1

[7] R. Bowen, Equilibrium states and the ergodic theory of Anosov diffeomorphisms, Lecture Notes in Mathematics, Springer-Verlag, Berlin-New York, (1975). 1

[8] R. Bowen, P. Walters, Expansive one-parameter flows, J. Differential Equations, 12 (1972), 180-193. 3

[9] J. Buzzi, Specification on the interval, Trans. Amer. Math. Soc., 349 (1997), 2737-2754. 3

[10] C.-K. Chu, K.-S. Koo, Recurrence and the shadowing property, Topology Appl., 71 (1996), 217-225. 1

[11] C. Conley, Isolated invariant sets and the Morse index, CBMS Regional Conference Series in Mathematics, American Mathematical Society, Providence, R.I., (1978).

[12] M. Denker, C. Grillenberger, K. Sigmund, Ergodic theory on compact spaces, Lecture Notes in Mathematics, SpringerVerlag, Berlin-New York, (1976).

[13] T. Gedeon, M. Kuchta, Shadowing property of continuous maps, Proc. Amer. Math. Soc., 115 (1992), 271-279.

[14] R.-B. Gu, The asymptotic average-shadowing property and transitivity for flows, Chaos Solitons Fractals, 41 (2009), 2234-2240. 1, 4

[15] R.-B. Gu, W.-J. Guo, The average-shadowing property and topological ergodicity for flows, Chaos Solitons Fractals, 25 (2005), 387-392. 1, 3, 3

[16] R.-B. Gu, Y.-Q. Sheng, Z.-J. Xia, The average-shadowing property and transitivity for continuous flows, Chaos Solitons Fractals, 23 (2005), 989-995. 1

[17] L.-F. He, Y.-H. Gao, F.-H. Yang, Some dynamical properties of continuous semi-flows having topological transitivity, Chaos Solitons Fractals, 14 (2002), 1159-1167. 1, 3, 4

[18] L.-F. He, G.-Z. Shan, Lifting and projecting continuous flows with the pseudo-orbit tracing property or the expansibility property, (Chinese); Acta Math. Appl. Sinica, 18 (1995), 1-7. 1, 3, 3, 4

[19] L.-F. He, Z.-H. Wang, Distal flows with pseudo-orbit tracing property, Chinese Sci. Bull., 39 (1994), 1936-1938. 1

[20] L.-F. He, Z.-H. Wang, H. Li, Continuous semiflows with the shadowing property, (Chinese); Acta Math. Appl. Sinica, 19 (1996), 297-303. 1, 3, 3, 4

[21] L.-F. He, Z. Zhang, Chaos in the semi-flows and its inverse limit systems, Acta Math. Sci., 17 (1997), 46-51. 3, 3, 3, 3

[22] B. Honary, A. Zamani Bahabadi, Orbital shadowing property, Bull. Korean Math. Soc., 45 (2008), 645-650. 1

[23] L.-L. Huang, D. Baleanu, G.-C. Wu, S.-D. Zeng, A new application of the fractional logistic map, Rom. J. Phys., 61 (2016), 1172-1179. 1

[24] L.-L. Huang, G.-C. Wu, M. M. Rashidi, W.-H. Luo, Chaos analysis of the nonlinear Duffing oscillators based on the new Adomian polynomials, J. Nonlinear Sci. Appl., 9 (2016), 1877-1881. 1

[25] K. Kato, Pseudo-orbits and stabilities of flows, Mem. Fac. Sci. Kochi Univ. Ser. A Math., 5 (1984), 45-62. 1

[26] M. Komuro, One-parameter flows with the pseudo-orbit tracing property, Monatsh. Math., 98 (1984), 219-253. 1

[27] P. Kościelniak, M. Mazur, Chaos and the shadowing property, Topology Appl., 154 (2007), 2553-2557. 1

[28] M. Kulczycki, P. Oprocha, Exploring the asymptotic average shadowing property, J. Difference Equ. Appl., 16 (2010), 1131-1140. 1, 3 
[29] M. Kulczycki, P. Oprocha, Properties of dynamical systems with the asymptotic average shadowing property, Fund. Math., 212 (2011), 35-52. 1

[30] S.-H. Lee, H.-J. Koh, S.-H. Ku, Investigation of the stability via shadowing property, J. Inequal. Appl., 2009 (2009), 12 pages. 1

[31] C.-P. Li, A new method of determining chaos-parameter-region for the tent map, Chaos Solitons Fractals, 21 (2004), 863-867. 3

[32] R.-S. Li, A note on shadowing with chain transitivity, Commun. Nonlinear Sci. Numer. Simul., 17 (2012), $2815-2823$. 2

[33] R.-S. Li, A note on stronger forms of sensitivity for dynamical systems, Chaos Solitons Fractals, 45 (2012), 753-758. 2

[34] R.-S. Li, A note on decay of correlation implies chaos in the sense of Devaney, Appl. Math. Model., 39 (2015), 6705-6710. $1,3,4$

[35] R.-S. Li, Y.-M. Shi, Stronger forms of sensitivity for measure-preserving maps and semiflows on probability spaces, Abstr. Appl. Anal., 2014 (2014), 10 pages. 2, 3.1

[36] M. Magdziarz, A. Weron, Ergodic properties of anomalous diffusion processes, Ann. Physics, 326 (2011), 2431-2443. 4

[37] J.-H. Mai, Pointwise recurrent dynamical systems with pseudo-orbit tracing property, Northeast. Math. J., 12 (1996), 73-78. 1

[38] J. Marklof, Entry and return times for semi-flows, Nonlinearity, 30 (2017), 810-824. 1

[39] T. K. S. Moothathu, Stronger forms of sensitivity for dynamical systems, Nonlinearity, 20 (2007), 2115-2126. 1, 3.15, $3.17,3,4$

[40] Y.-X. Niu, S.-B. Su, On strong ergodicity and chaoticity of systems with the asymptotic average shadowing property, Chaos Solitons Fractals, 44 (2011), 429-432. 1, 4

[41] S. Y. Pilyugin, Sets of dynamical systems with various limit shadowing properties, J. Dynam. Differential Equations, 19 (2007), 747-775. 1

[42] S. Y. Pilyugin, K. Sakai, $C^{0}$ transversality and shadowing properties, translated from Tr. Mat. Inst. Steklova, Din. Sist. i Optim., 256 (2007), 305-319, Proc. Steklov Inst. Math., 256 (2007), 290-305. 1

[43] K. Sakai, Diffeomorphisms with the average-shadowing property on two-dimensional closed manifolds, Rocky Mountain J. Math., 3 (2000), 1129-1137. 1

[44] K. Sakai, Shadowing properties of L-hyperbolic homeomorphisms, Topology Appl., 112 (2001), 229-243. 1

[45] K. Sakai, Various shadowing properties for positively expansive maps, Topology Appl., 131 (2003), 15-31. 1, 3.13

[46] S. Smale, Differentiable dynamical systems, Bull. Amer. Math. Soc., 73 (1967), 747-817. 1

[47] R. F. Thomas, Stability properties of one-parameter flows, Proc. London Math. Soc., 45 (1982), 479-505. 3.20

[48] D. Thomine, Variations on a central limit theorem in infinite ergodic theory, Ergodic Theory Dynam. Systems, 35 (2015), 1610-1657. 1

[49] D. Thomine, Local time and first return time for periodic semi-flows, Israel J. Math., 215 (2016), 53-98. 1, 3

[50] X.-J. Tong, Design of an image encryption scheme based on a multiple chaotic map, Commun. Nonlinear Sci. Numer. Simul., 18 (2013), 1725-1733. 3

[51] P. Walters, On the pseudo-orbit tracing property and its relationship to stability, The structure of attractors in dynamical systems, Proc. Conf., North Dakota State Univ., Fargo, N.D., (1977), Lecture Notes in Math., Springer, Berlin, 668 (1978), 231-244. 1

[52] P. Walters, An introduction to ergodic theory, Graduate Texts in Mathematics, Springer-Verlag, New York-Berlin, (1982). 3.19

[53] G.-C. Wu, D. Baleanu, Chaos synchronization of the discrete fractional logistic map, Signal Process., 102 (2014), 96-99. 1

[54] G.-C. Wu, D. Baleanu, Discrete fractional logistic map and its chaos, Nonlinear Dynam., 75 (2014), 283-287. 1

[55] G.-C. Wu, D. Baleanu, H.-P. Xie, F.-L. Chen, Chaos synchronization of fractional chaotic maps based on the stability condition, Phys. A, 460 (2016), 374-383. 1

[56] S.-L. Wu, G.-C. Wu, Nonoverlapping Schwarz waveform relaxation algorithm for a class of time-fractional heat equations, Fund. Inform., 151 (2017), 231-240. 1

[57] X.-H. Yan, L.-F. He, Two remarks on sensitive dependence of semi-dynamical systems, Southeast Asian Bull. Math., 32 (2008), 393-398. 3

[58] R.-S. Yang, The pseudo-orbit tracing property and chaos, (Chinese); Acta Math. Sinica (Chin. Ser.), 39 (1996), $382-386$. 1

[59] R. S. Yang, S. L. Shen, Pseudo-orbit-tracing and completely positive entropy, (Chinese); Acta Math. Sinica (Chin. Ser.), 42 (1999), 99-104. 1 